\title{
Performance Diagnóstica da FFR por Angiotomografia de Coronárias através de Software Baseado em Inteligência Artificial
}

\author{
Diagnostic Performance of a Machine Learning-Based CT-Derived FFR in Detecting Flow-Limiting Stenosis
}

Thamara Carvalho Morais, ${ }^{1,2}$ Antonildes Nascimento Assunção-Jr, ${ }^{1,2}$ Roberto Nery Dantas Júnior, ${ }^{1,2}$ Carla Franco Grego da Silva, ${ }^{1}$ Caroline Bastida de Paula, ${ }^{1}$ Roberto Almeida Torres, ${ }^{1,2}$ Targago Augusto Magalhães, ${ }^{1,2,3}$ César Higa Nomura, ${ }^{1,2}$ Luiz Francisco Rodrigues de Ávila, 1,2 José Rodrigues Parga Filho ${ }^{1,2}$

Hospital Sírio-libanês, ${ }^{1}$ São Paulo, SP - Brasil

Universidade de São Paulo Faculdade de Medicina - CDI - InCor/HCFMUSP - Departamento de Imagem Cardiovascular, ${ }^{2}$ São Paulo, SP - Brasil Complexo Hospital de Clínicas da Universidade Federal do Paraná (CHC-UFPR) - Cardiovascular CT/MR, ${ }^{3}$ Curitiba, PR - Brasil

\section{Resumo}

Fundamento: A quantificação não invasiva da reserva fracionada de fluxo miocárdico ( $F F R_{\mathrm{TC}}$ ) através de software baseado em inteligência artificial em versão mais atualizada e tomógrafo de última geração ( 384 cortes) apresenta elevada performance na detecção de isquemia coronariana.

Objetivos: Avaliar o desempenho diagnóstico da $\mathrm{FFR}_{\mathrm{TC}}$ na detecção de doença arterial coronariana (DAC) significativa em relação ao FFRi, em tomógrafos de gerações anteriores (128 e 256 cortes).

Métodos: Estudo retrospectivo com pacientes encaminhados à angiotomografia de artérias coronárias (TCC) e cateterismo (FFRi). Foram utilizados os tomógrafos Siemens Somatom Definition Flash (256 cortes) e AS+ (128 cortes). $\mathrm{A} \mathrm{FFR}_{\mathrm{TC}}$ e a área luminal mínima (ALM) foram avaliadas em software (cFFR versão 3.0.0, Siemens Healthineers, Forchheim, Alemanha). DAC obstrutiva foi definida como TCC com redução luminal $\geq 50 \%$ e DAC funcionalmente obstrutiva como FFRi $\leq 0,8$. Todos os valores de $p$ reportados são bicaudais; e quando $<0,05$, foram considerados estatisticamente significativos.

Resultados: Noventa e três pacientes consecutivos (152 vasos) foram incluídos. Houve boa concordância entre $\mathrm{FFR}_{\mathrm{TC}}$ e FFRi, com mínima superestimação da $\mathrm{FFR}_{\mathrm{TC}}$ (viés: -0,02; limites de concordância: 0,14 a 0,09). Diferentes tomógrafos não modificaram a relação entre $\mathrm{FFR}_{\mathrm{TC}}$ e FFRi (p para interação $\left.=0,73\right)$. A $\mathrm{FFR}_{\mathrm{TC}}$ demonstrou performance significativamente superior à classificação visual de estenose coronariana (AUC 0,93 vs. 0,61, p <0,001) e à ALM (AUC 0,93 vs. $0,75, \mathrm{p}<0,001$ ) reduzindo o número de casos falso-positivos. $\mathrm{O}$ melhor ponto de corte para a $\mathrm{FFR}_{\mathrm{TC}}$ utilizando um índice de Youden foi de 0,85 (sensiblidade, 87\%; especificidade, 86\%; VPP, $73 \%$; NPV, 94\%), com redução de falso-positivos.

Conclusão: FFR $_{\mathrm{TC}}$ baseada em inteligência artificial, em tomógrafos de gerações anteriores (128 e 256 cortes), apresenta boa performance diagnóstica na detecção de DAC, podendo ser utilizada para reduzir procedimentos invasivos.

Palavras-chave: Reserva Fracionada de Fluxo Miocárdico; Doença Arterial Coronariana; Tomografia Computadorizada; Isquemia Miocárdica; Aprendizado de Máquina.

\footnotetext{
Abstract

Background: The non-invasive quantification of the fractional flow reserve (FFRCT) using a more recent version of an artificial intelligence-based software and latest generation CT scanner (384 slices) may show high performance to detect coronary ischemia.

Objectives: To evaluate the diagnostic performance of FFRCT for the detection of significant coronary artery disease (CAD) in contrast to invasive FFR (iFFR) using previous generation CT scanners (128 and 256- detector rows).

Methods: Retrospective study with patients referred to coronary artery CT angiography (CTA) and catheterization (iFFR) procedures. Siemens Somatom Definition Flash (256-detector rows) and AS+ (128-detector rows) CT scanners were used to acquire the images. The FFRCT and the minimal lumen area (MLA) were evaluated using a dedicated software (CFFR version 3.0.0, Siemens Healthineers, Forchheim, Germany). Obstructive CAD was defined as CTA lumen reduction $\geq 50 \%$, and flow-limiting stenosis as iFFR $\leq 0.8$. All reported $P$ values are two-tailed, and when $<0.05$, they were considered statistically significant.
}

Correspondência: Thamara Carvalho Morais •

Hospital Sírio-libanês - Rua Adma Jafet, 91. CEP 01308-050, São Paulo, SP - Brasil

E-mail: thamaramorais@hotmail.com

Artigo recebido em 26/05/2019, revisado em 11/02/2020, aceito em 16/03/2020

DOI: https://doi.org/10.36660/abc.20190329 
Results: Ninety-three consecutive patients (152 vessels) were included. There was good agreement between FFRCT and iFFR, with minimal FFRCT overestimation (bias: -0.02; limits of agreement:0.14-0.09). Different CT scanners did not modify the association between FFRCT and FFRi ( $p$ for interaction =0.73). The performance of FFRCT was significantly superior compared to the visual classification of coronary stenosis (AUC 0.93vs.0.61, $p<0.001$ ) and to MLA (AUC 0.93vs.0.75, p<0.001), reducing the number of false-positive cases. The optimal cut-off point for FFRCT using a Youden index was 0.85 (87\% Sensitivity, 86\% Specificity, 73\% PPV, 94\% NPV), with a reduction of false-positives.

Conclusion: Machine learning-based FFRCT using previous generation CT scanners (128 and 256-detector rows) shows good diagnostic performance for the detection of CAD, and can be used to reduce the number of invasive procedures.

Keywords: Myocardial Fractional Flow Reserve, Coronary Artery Disease, Computed Tomography, Myocardial Ischemic, Machine Learning

Full texts in English - http://www.arquivosonline.com.br

\section{Introdução}

De acordo com as diretrizes clínicas mais recentes, ${ }^{1-3}$ o manejo da doença arterial coronariana (DAC) crônica e sintomática pode ser guiado por testes adicionais para avaliação anatômica (extensão, severidade, morfologia) ou funcional (função ventricular, presença/extensão de isquemia), com algumas evidências apontando superioridade da avaliação funcional sobre anatômica. ${ }^{4-6}$

Para este propósito, sobretudo em pacientes com probabilidade pré-teste intermediária para DAC obstrutiva, a angiotomografia computadorizada de artérias coronárias (TCC) vem se destacando dentre os vários testes não invasivos como método robusto para descartar DAC obstrutiva, por seu elevado valor preditivo negativo. ${ }^{7}$ Particularmente em estenoses moderadas (50\% a 69\%), a quantificação não invasiva da reserva fracionada de fluxo miocárdico $\left(\mathrm{FFR}_{\mathrm{TC}}\right)$ pode ajudar na correta discriminação de quais destas estão associadas à isquemia. ${ }^{8}$ Estudos recentes demonstraram que a TCC tem elevada acurácia para identificar isquemia miocárdica por meio da quantificação não invasiva da FFRTC quando comparada ao padrão-ouro, a FFR invasiva pelo cateterismo cardíaco (FFRi). ${ }^{8-10}$

A grande restrição do uso da $\mathrm{FFR}_{\mathrm{TC}}$ na prática clínica têm sido sua baixa disponibilidade, especialmente devido à necessidade de software específico que requeria supercomputadores em grandes centros internacionais, encarecendo e prolongando substancialmente o processo. ${ }^{8}$ Recentemente, um protótipo não comercial de software (disponível para computadores pessoais de configuração-padrão) que utiliza ferramentas de inteligência artificial - rede neural convolucional (deep learning) - para avaliação da $\mathrm{FFR}_{\mathrm{TC}}$ foi testado por Rother et al.,11 Quando comparado com FFRi, a FFR ${ }_{\mathrm{TC}}$ calculada por esse software demonstrou elevada acurácia para detecção de isquemia, com significativa redução no tempo de execução do seu cálculo quando comparado com modelos já existentes que utilizam supercomputadores. ${ }^{8}$ Deve-se ressaltar, contudo, que este trabalho utilizou apenas imagens de um tomógrafo de última geração (Siemens Somatom Force - 384 cortes). Uma vez que esse software se propõe a calcular a FFR em imagens adquiridas em tomógrafos com diferentes tecnologias, objetivamos utilizá-lo para investigar a acurácia diagnóstica da FFR ${ }_{\mathrm{TC}}$ em tomógrafos de gerações anteriores, em comparação com FFRi, com qualidade de imagens que podem potencialmente afetar os resultados do algoritmo utilizado no software. Esse estudo também comparou a acurácia diagnóstica da FFR ${ }_{\mathrm{TC}}$ Com a avaliação anatômica isolada pela TCC.

\section{Métodos}

\section{População do estudo}

Retrospectivamente, este estudo incluiu pacientes com sintomas sugestivos de DAC significativa encaminhados para TCC. Após os achados desse exame, foram encaminhados por decisão clínica para o cateterismo cardíaco (com intervalo menor de 30 dias) e que realizaram análise de FFRi no Hospital Sírio-Libanês (São Paulo-SP), entre janeiro de 2014 e fevereiro de 2018. No total, houve 17 exclusões: 14 por fatores limitantes para o cálculo da $\mathrm{FFR}_{\mathrm{TC}}$ descriminados pelo fabricante da ferramenta (8 por lesão de tronco de coronária esquerda [TCE], óstios ou bifurcações; 6 pela presença de stent); e 3 por imagem com qualidade insuficiente devido a calcificação excessiva e significativos artefatos de movimento. Durante o pós-processamento das imagens, não houve exclusão de pacientes por impossibilidade técnica de o software realizar a quantificação da FFR $\mathrm{TC}_{\text {C }}$ Ressalta-se que as recomendações do fabricante com relação a lesões de TCE, óstios ou bifurcações têm sido seguidas também por outros autores, ${ }^{11}$ e parecem estar relacionadas ao reconhecimento limitado das bordas anatômicas nesses cenários. Esse estudo foi aprovado pelo Comitê de Ética em Pesquisa do Hospital Sírio-Libanês.

\section{Aquisição de imagens da TCC}

As imagens foram obtidas utilizando-se os tomógrafos Siemens Somatom Definition Flash de 256 cortes (resolução temporal - TR - de 75ms; resolução espacial - SR - de 0,30mm) e Somaton Definition AS+ de 128 cortes (TR de150ms; SR de 0,30mm) (Siemens Healthineers, Forchheim, Alemanha). O preparo do paciente seguiu recomendações das diretrizes atuais, incluindo jejum de $4 \mathrm{~h}$, punção venosa com jelco calibre 18G, preferencialmente à direita, e monitoramento eletrocardiográfico contínuo. ${ }^{12}$ Sempre que necessário, para controle da frequência cardíaca (FC), foi administrado betabloqueador (tartarato de metoprolol 50 a $100 \mathrm{mg}$ oral $1 \mathrm{~h}$ antes do exame e/ou 5 a $20 \mathrm{mg}$ endovenoso poucos minutos antes da aquisição), objetivando-se manter a FC em torno de 55 a $60 \mathrm{bpm}$. Todos os pacientes receberam ainda nitrato sublingual (isordil 2,5mg) poucos minutos antes da aquisição, exceto nos casos de hipotensão sintomática ou uso de inibidores de fosfodiesterase tipo 5 (de acordo com o tempo de ação de cada fármaco).

A aquisição das imagens foi planejada após a realização de teste bolus para cálculo do tempo de pico do contraste 
na aorta, com volume de 10 a $15 \mathrm{~mL}$, seguido de 30 a $50 \mathrm{~mL}$ de soro fisiológico a 4,5 a $5,5 \mathrm{~mL} / \mathrm{s}$. As imagens foram adquiridas utilizando o modo Flash (no tomógrafo Definition Flash) ou de forma retrospectiva (em ambos os tomógrafos) com acoplamento eletrocardiográfico na diástole (55\% a $75 \%$ do RR), corrente do tubo de 100 a 120kVp (ajustado pelo índice de massa corporal [IMC] individual), tempo de rotação de 0,28 (Flash)/0,33 (AS+) segundos, 160 a 320mAs e espessura de corte de $0,6 / 0,3 \mathrm{~mm}$. A infusão de contraste iodado Optiray 350 (loversol 350mg/mL, Mallinckrodt-EUA) utilizou os mesmos parâmetros do teste bolus (60 a 90mL).

\section{Análise de imagens da TCC e FFRct}

A análise das imagens da TCC foi realizada por meio do software Syngo.via (Siemens Healthineers, Forchheim, Germany). Após a escolha de imagens de melhor qualidade técnica, foram feitas avaliações da árvore coronariana em reformatação tridimensional, multiplanar e curva (vessel probe), com quantificação do grau de estenose e composição predominante da placa, quando presente (não calcificada, calcificada e mista). A quantificação da redução luminal foi realizada conforme recomendação da Sociedade de Tomografia Computadorizada Cardiovascular: ${ }^{13}$ ausente, mínima (<25\%), discreta (25\% a 49\%), moderada (50\% a $69 \%)$, importante (70\% a 99\%) e oclusão (100\%). As estenoses foram também classificadas como obstrutivas ( $\geq 50 \%)$ e não obstrutivas $(<50 \%)$ pela TCC.

O pós-processamento da $F_{F R}$ foi feito na mesma série em que foi realizada a análise visual anatômica (descrita anteriormente), utilizando a plataforma Frontier e o protótipo não comercial do software cFFR, versão 3.0 (Siemens Healthineers, Forchheim, Alemanha) por um médico experiente em imagem cardiovascular ( $>4$ anos).

No cálculo da $\mathrm{FFR}_{\mathrm{TC}}$, inicialmente, foi realizada a detecção automática da linha central e dos contornos do lúmen das artérias coronárias, que foram revisados e corrigidos pelo médico especialista, quando necessário. Na sequência, o médico definiu o limite superior e inferior de todas as placas dos vasos que tiveram os valores de FFRi calculados no cateterismo.

Para a identificação correta do local em que se calculou FFRi, o médico hemodinamicista localizou o ponto de interesse nas imagens da fluoroscopia e documentou usando referências anatômicas (ramos das coronárias) e também um modelo de segmentação coronariana sugerido pela Society of Cardiovascular Computed Tomography (SCCT). O médico especialista em TCC utilizou essa documentação para selecionar a mesma localização anatômica das placas que tiverem a $\mathrm{FFR}_{\mathrm{TC}}$ calculada.

O software cFFR calcula valores de FFR para toda a árvore coronariana com diâmetro $\geq 1,5 \mathrm{~mm}$ e calcula automaticamente o valor ALM de cada placa delimitada. As etapas para cálculo da $\mathrm{FFR}_{\mathrm{TC}}$ estão demonstradas na Figura 1. Esse software foi desenvolvido com novas ferramentas de inteligência artificial, utilizando-se técnicas de aprendizado de máquina (deep learning). Todos eles foram instalados em computador padrão para laudos de radiologia. O tempo total de pós-processamento de todas as etapas foi em torno de 10 minutos.

\section{Cateterismo cardíaco e análise de FFRi}

O cateterismo cardíaco foi realizado via radial ou femoral, utilizando cateteres diagnósticos de 6 ou 7 french (F). Para o cálculo de FFRi, o grau de estenose foi avaliado visualmente pelo hemodinamicista, utilizando-se pelo menos duas projeções ortogonais. As medidas de FFR foram realizadas com cateteresguia de 6 ou 7F. Nitroglicerina intracoronária $(0,2 \mathrm{mg})$ foi injetada em todos os pacientes antes dos angiogramas. Um fio-guia de monitoramento de pressão foi posicionado distal à lesão-índice, e as pressões médias foram registradas no momento em que ficam estáveis. Adenosina intracoronariana foi injetada manualmente através do cateter-guia, em injeção de bolus de $80 \mu \mathrm{g}$ (artéria coronária esquerda) ou 40 $\mu$ g (coronária direita) em $10 \mathrm{~mL}$ de soro fisiológico. Após sua administração, o menor valor de FFR estável durante o estado estacionário hiperêmico foi registrado. Esse valor corresponde à razão entre a pressão coronariana média distal à estenose e a pressão aórtica média no momento da hiperemia induzida farmacologicamente.

A exata posição do sensor de medida de FFRi foi documentada pelo hemodinamicista no laudo, e esta documentação foi utilizada pelo médico especialista em TCC para a medida da $\mathrm{FFR}_{\mathrm{TC}}$ na mesma localização anatômica.

\section{Análise estatística}

Análises descritivas foram expressas como frequência (porcentagem) para variáveis categóricas e como média \pm desvio padrão para variáveis contínuas. A distribuição das variáveis contínuas foi avaliada por meio visual através de gráficos QQ e checada pelo teste de Shapiro-Wilk. Comparações entre as variáveis contínuas encontradas na TCC e no cateterismo (grau de estenose coronariana, FFR) dos pacientes foram realizadas pelo teste t de Student para amostras pareadas. Da mesma forma, correlação entre essas variáveis foi realizada por meio de correlação de Pearson.

Concordância entre $\mathrm{FFR}_{\mathrm{TC}}$ e FFRi foi determinada por análise de Bland-Altman. Assumindo FFRi $\leq 0,8$ como padrão-ouro para presença de isquemia, a performance diagnóstica da $\mathrm{FFR}_{\mathrm{TC}}$ e de outros parâmetros anatômicos da TCC foi avaliada por meio do cálculo de sensibilidade, especificidade, valor preditivo positivo (VPP) e valor preditivo negativo (VPN). Além disso, a área sob a curva ROC (AUC) para detecção de lesões coronarianas associadas com isquemia foi calculada, e comparações entre as AUC foram realizadas de acordo com o método descrito por DeLong et al., ${ }^{14} \mathrm{O}$ melhor ponto de corte da FFR TC para detecção de isquemia (FFRi $\leq 0,8$ ) foi calculado utilizando-se o índice de Youden, que corresponde àquele com maior valor na equação (sensibilidade [Sens] + especificidade [Espe] - 1). ${ }^{15}$ Ressalta-se que o grau de estenose foi explorado como variável contínua e categórica (DAC obstrutiva, $>50 \%$, ou não). A escolha pela forma categórica para inclusão no modelo se baseou em se tratar de um limiar clinicamente acionável para tomadas de decisões clínicas subsequentes.

Considerando a potencial correlação entre múltiplos vasos no mesmo indivíduo, o método de equações de estimação generalizadas com estrutura de correlação permutável foi usado para comparar amostras pareadas em um nível por-vaso. As análises estatísticas foram realizadas no software $\mathrm{R}$ ( $R$ Foundation for Statistical Computing, Viena, Áustria). Todos os valores de $p$ 


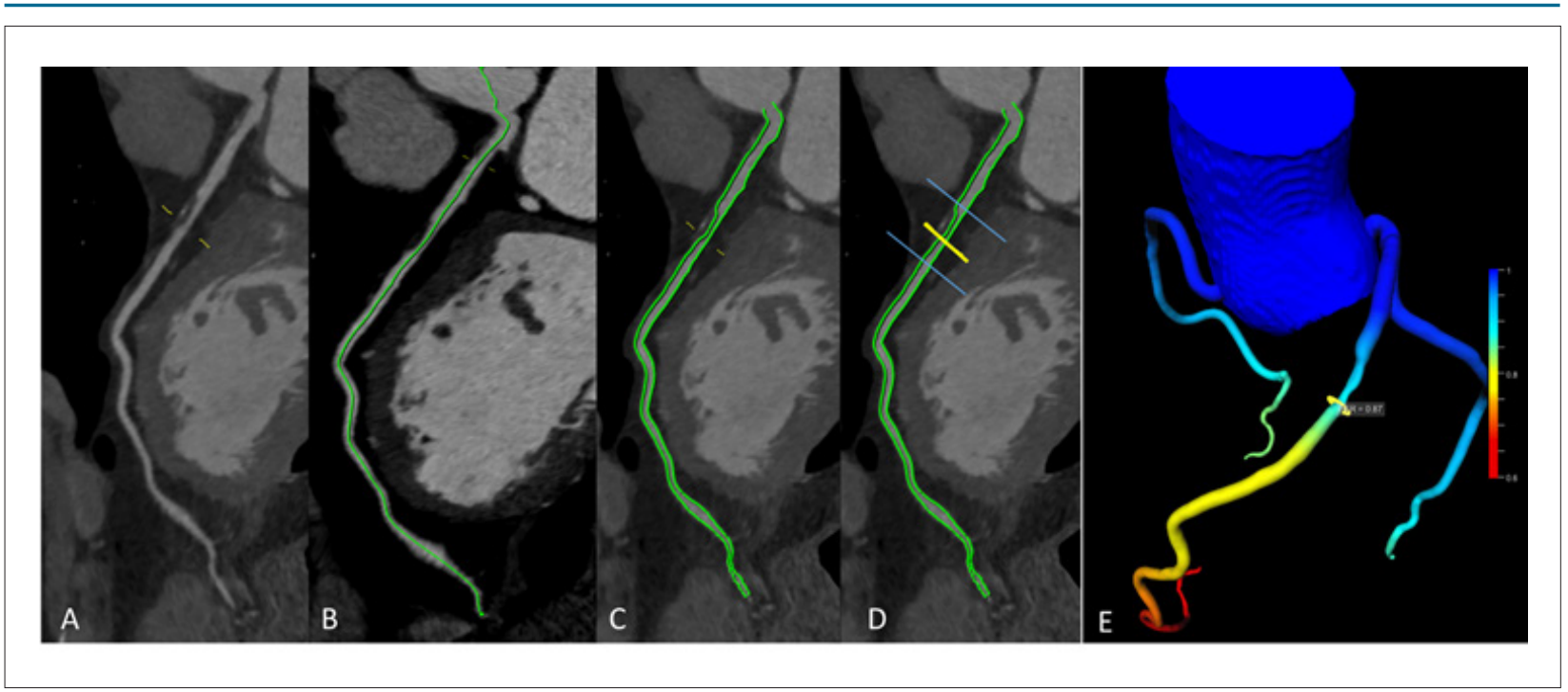

Figura 1 - Etapas para cálculo da $F F R_{T C}$ utilizando o software cFFR. A) Detecção visual da lesão coronariana. Definição do centerline B) e contornos luminais C) pelo software CFFR D) definição dos limites da lesão e do ponto de maior redução luminal pelo operador. E) Resultado do FFR no ponto de maior redução luminal demonstrado na árvore coronariana (após determinar centerline e contornos luminais nas três principais artérias coronárias: $D A, C x$ e $C D)$.

reportados são bicaudais e, quando $<0,05$, foram considerados estatisticamente significativos.

\section{Resultados}

\section{Características dos pacientes e das placas}

Noventa e três pacientes foram incluídos no estudo, com um total de 152 vasos. Cinquenta pacientes (54\%) realizaram a TCC em tomógrafo Flash (256 colunas de detectores) e 43 (46\%) em tomógrafo AS+ (128 colunas de detectores). A FC média nas aquisições foi de $58 \pm 8 \mathrm{bpm}$.

Setenta e quatro pacientes (80\%) apresentavam DAC obstrutiva (estenose $>50 \%$ ) na TCC, sendo 48 com estenose moderada (50\% a 69\%) e 26 importante $(>70 \%)$. Na análise por-vaso, as placas foram mais frequentemente mistas (70\%), com localização mais comum (73\%) na coronária descendente anterior (DA) e com ALM média de 3,2 \pm $1,6 \mathrm{~mm}^{2}$. Características clínicas e tomográficas dos pacientes estão expostas nas Tabelas 1 e 2, respectivamente.

\section{Comparação de $\mathrm{FFR}_{\mathrm{TC}}$ com FFRi}

Houve forte correlação entre os valores da FFR $\mathrm{TC}_{\mathrm{TC}}$ e FFRi $(r$ $=0,73, p<0,001$ ) (Figura 2). Na média, os valores da FFR foram discretamente superiores aos encontrados em FFRi $(0,88 \pm 0,08$ vs. $0,86 \pm 0,08, p=0,02)$, erro sistemático que se confirma na análise de Bland-Altman (viés de -0,02 com intervalo de confiança de $-0,14$ a 0,09) (ver Figura 2). O tipo de tomógrafo utilizado não mudou a relação entre FFRi e $\mathrm{FFR}_{\mathrm{TC}}$ (p-valor para interação de 0,73 ).

\section{Detecção de isquemia}

Para identificação de lesões coronarianas obstrutivas com limitação de fluxo (FFRi $\leq 0,8$ como padrão-ouro), a
$\mathrm{FFR}_{\mathrm{TC}}$ demonstrou performance significativamente superior à classificação visual isolada de obstrução coronariana (AUC 0,93 vs. 0,61, p <0,001) e à ALM pela TCC (AUC 0,93 vs. 0,75, $\mathrm{p}<0,001$ ) (Figura 3). O melhor ponto de corte (com menor número de resultados falsos) para a $\mathrm{FFR}_{\mathrm{TC}}$ definido por meio do índice de Youden, para discriminação de lesão com ou sem isquemia, foi 0,85 , que apresentou valores de sensibilidade de $87 \%$, especificidade de $86 \%$, VPP de $73 \%$ e VPN de $94 \%$ nesse ponto de corte (Figura 4). Tais métricas de performance utilizando-se esse ponto de corte $(0,85)$ foram discretamente superiores analisando-se apenas as placas com redução luminal moderada ( $50 \%$ a $69 \%, \mathrm{n}=95$ ), com sensibilidade de $89 \%$, especificidade de 91\%, VPP de 74\%, VPN de $97 \%$. Das 152 lesões avaliadas, 3 (2\%) foram falso-positivas e 18 (12\%), falsonegativas, utilizando-se o ponto de corte tradicional (FFR $\leq 0,80)$. Por meio do ponto de corte mais elevado (FFR TC $\left._{2}<0,85\right)$, $12(7 \%)$ foram falso-positivas e 9 (6\%), falso-negativas.

Ao avaliarmos o grau de redução luminal, as placas visualmente consideradas moderadas (50\% a 69\%) tiveram um número de falso-positivos de 86 (56\%) em relação ao padrãoouro (FFRi $\leq 0,8)$, enquanto as placas com redução luminal visual importante ( $\geq 70 \%$ ) tiveram um número de falso-positivos de 23 (15\%), o que, para esta última, representa uma magnitude 50\% maior em relação aos resultados da $\mathrm{FFR}_{\mathrm{TC}}<0,85$ (15\% vs. 7\%).

\section{Discussão}

A análise da FFR ${ }_{\mathrm{TC}}$ através de software baseado em aprendizado de máquina demonstrou boa concordância em relação à medida de FFRi, destacando-se que o pós-processamento das imagens de TCC foi feito em computadores-padrão do próprio hospital e com tempo de pós-processamento em torno de 10min. Com relação à performance diagnóstica, mesmo em tomógrafos de gerações anteriores, a FFR $\mathrm{TC}_{\mathrm{C}}$ foi superior à avaliação anatômica isolada tanto na observação de estenoses coronarianas como 


\begin{tabular}{|c|c|}
\hline Variáveis & $n=93$ \\
\hline Idade, anos* & $64 \pm 11$ \\
\hline Sexo masculino, n (\%) & $70(75)$ \\
\hline Hipertensão, n (\%) & $54(58)$ \\
\hline Dislipidemia, n (\%) & $45(48)$ \\
\hline Diabetes, n (\%) & $24(26)$ \\
\hline Tabagismo, n (\%) & $7(8)$ \\
\hline $\mathrm{IMC}, \mathrm{kg} / \mathrm{m}^{2 *}$ & $28 \pm 4$ \\
\hline $\mathrm{FC}, \mathrm{bpm}^{*}$ & $58 \pm 8$ \\
\hline
\end{tabular}

Tabela 2 - análise do FFRTC e FFRi

\begin{tabular}{|c|c|}
\hline Por paciente & $n=93$ \\
\hline Estenose $\geq 50 \%, \mathrm{n}(\%)$ & $74(80)$ \\
\hline Estenose $50-69 \%, \mathrm{n}(\%)$ & $48(52)$ \\
\hline Estenose $\geq 70 \%, \mathrm{n}(\%)$ & $26(28)$ \\
\hline $\mathrm{FFR}_{\mathrm{TC}} \leq 0,8, \mathrm{n}(\%)$ & $32(34)$ \\
\hline $\mathrm{FFRi} \leq 0,8, \mathrm{n}(\%)$ & $39(42)$ \\
\hline Por vaso & $n=152$ \\
\hline \multicolumn{2}{|l|}{ Localização } \\
\hline $\mathrm{DA}, \mathrm{n}(\%)$ & $111(73)$ \\
\hline$C x, n(\%)$ & $26(17)$ \\
\hline $\mathrm{CD}, \mathrm{n}(\%)$ & $16(10)$ \\
\hline Estenose $\geq 50 \%, \mathrm{n}(\%)$ & $124(82)$ \\
\hline Estenose $50-69 \%, \mathrm{n}(\%)$ & $95(63)$ \\
\hline Estenose $\geq 70 \%, \mathrm{n}(\%)$ & $29(19)$ \\
\hline $\mathrm{ALM}, \mathrm{mm}^{2 *}$ & $3,2 \pm 1,6$ \\
\hline \multicolumn{2}{|l|}{ Morfologia } \\
\hline Calcificada, n (\%) & $16(10)$ \\
\hline Mista, n (\%) & $106(70)$ \\
\hline Não calcificada, n (\%) & $30(20)$ \\
\hline $\mathrm{FFR}_{\mathrm{TC}}{ }^{*}$ & $0,88 \pm 0,08$ \\
\hline $\mathrm{FFR}_{\mathrm{TC}} \leq 0,8, \mathrm{n}(\%)$ & $32(21)$ \\
\hline FFRi* & $0,86 \pm 0,08$ \\
\hline $\mathrm{FFRi} \leq 0,8, \mathrm{n}(\%)$ & $47(31)$ \\
\hline
\end{tabular}

no cálculo da ALM, reduzindo significativamente o número de falso-positivos.

Em linha com o demonstrado por Rother et al., ${ }^{11}$ retrospectivamente em coorte com 71 pacientes e utilizando a mesma versão de software desse estudo (cFFR versão 3.0), ${ }^{11}$ a FFR ${ }_{\mathrm{TC}}$ apresentou considerável concordância com a medida de FFRi, com mínima superestimação. Esses resultados estão em desencontro com versões anteriores desse mesmo software (cFFR versão 1.4), ${ }^{16-19}$ em que uma subestimação foi descrita, e provavelmente reflete mudanças do algoritmo com a atualização do software.

Apesar de comparável com os três principais estudos multicêntricos até hoje publicados (DISCOVER-FLOW, DeFacto and NXT), ${ }^{8-10}$ há que se realçar que o limite de concordância do nosso estudo foi mais largo na análise de Bland-Altman $(\sim 0,20)$, que significa menor repetibilidade do método, em comparação com o observado por Rother et al., ${ }^{11}$ Como, na média, os pacientes do nosso estudo apresentaram uma FC média $<60$ bpm, o que reflete uma boa qualidade das imagens em geral, acreditamos que o desempenho superior daquele estudo pode ser explicado, em parte, pela utilização de um tomógrafo com resolução espacial $20 \%$ superior $(0,3$ vs. $0,24 \mathrm{~mm}$ ), além do uso de um moderno algoritmo de reconstrução (ADMIRE). Esses fatores podem ter levado a uma melhor detecção dos contornos coronarianos (linha central e lúmen) por aquele estudo, com consequente melhora dos resultados. Outra justificativa que não pode ser descartada seria a maior experiência do observador daquele centro com a nova versão de cFFR.

Com relação ao poder de discriminação de estenoses coronarianas com/sem limitação de fluxo, a FFR TC foi superior em comparação com a avaliação isolada anatômica da TCC, tanto qualitativamente (classificação visual de DAC obstrutiva) quanto quantitativamente (ALM). Utilizando-se um ponto de corte de 0,85 para a FFR $\mathrm{TC}^{\prime}$ os VPN e VPP foram comparáveis com os de outras coortes que utilizaram esse software. ${ }^{16-19}$ Além disso, ressaltamos os seguintes aspectos: 1) a performance da $\mathrm{FFR}_{\mathrm{TC}}$ foi melhor nos casos com lesões moderadas (50\% a 69\%); 2) a FFR levou a uma redução de mais de $50 \%$ dos casos falso-positivos quando usada somente a avaliação anatômica de DAC grave $(\geq 70 \%)$. Esses achados apresentam grande relevância na prática clínica, uma vez que lesões moderadas na TCC são relativamente frequentes e, muitas vezes, esses pacientes são encaminhados para exames adicionais. ${ }^{20}$ De fato, a oportunidade de redução global de encaminhamento desnecessário para cateterismo pode ser ainda maior utilizandose essa nova ferramenta de $\mathrm{FFR}_{\mathrm{T} \mathrm{C}^{\prime}}$ uma vez que apenas $42 \%$ dos nossos pacientes apresentaram FFRi $<0,8$.

Por fim, destaca-se o rápido pós-processamento de imagens desse novo software com base em tecnologia de aprendizado de máquina (deep learning). Nos softwares pioneiros ${ }^{8-10}$ que utilizam algoritmos de dinâmica de fluidos, o cálculo da $\mathrm{FFR}_{\mathrm{TC}}$ leva de 1 a 4 h de processamento, e é realizado em supercomputadores localizados apenas em centros específicos dos EUA (sede na Califórnia), Londres e Tóquio. Além do elevado custo, no geral, isso demanda cerca de $24 \mathrm{~h}$ para a obtenção dos resultados e necessidade de enviar imagens DICOM para fora de ambientes da instituição. Portanto, esse novo software poderá promover impacto real na prática clínica no cuidado do paciente com DAC.

\section{Limitações}

Trata-se de um estudo retrospectivo, unicêntrico, com uma população de estudo relativamente pequena e predominantemente apresentando DAC obstrutiva. Ao seguirmos as recomendações de aplicabilidade da ferramenta pelo fabricante, foram excluídos os pacientes com estenose 


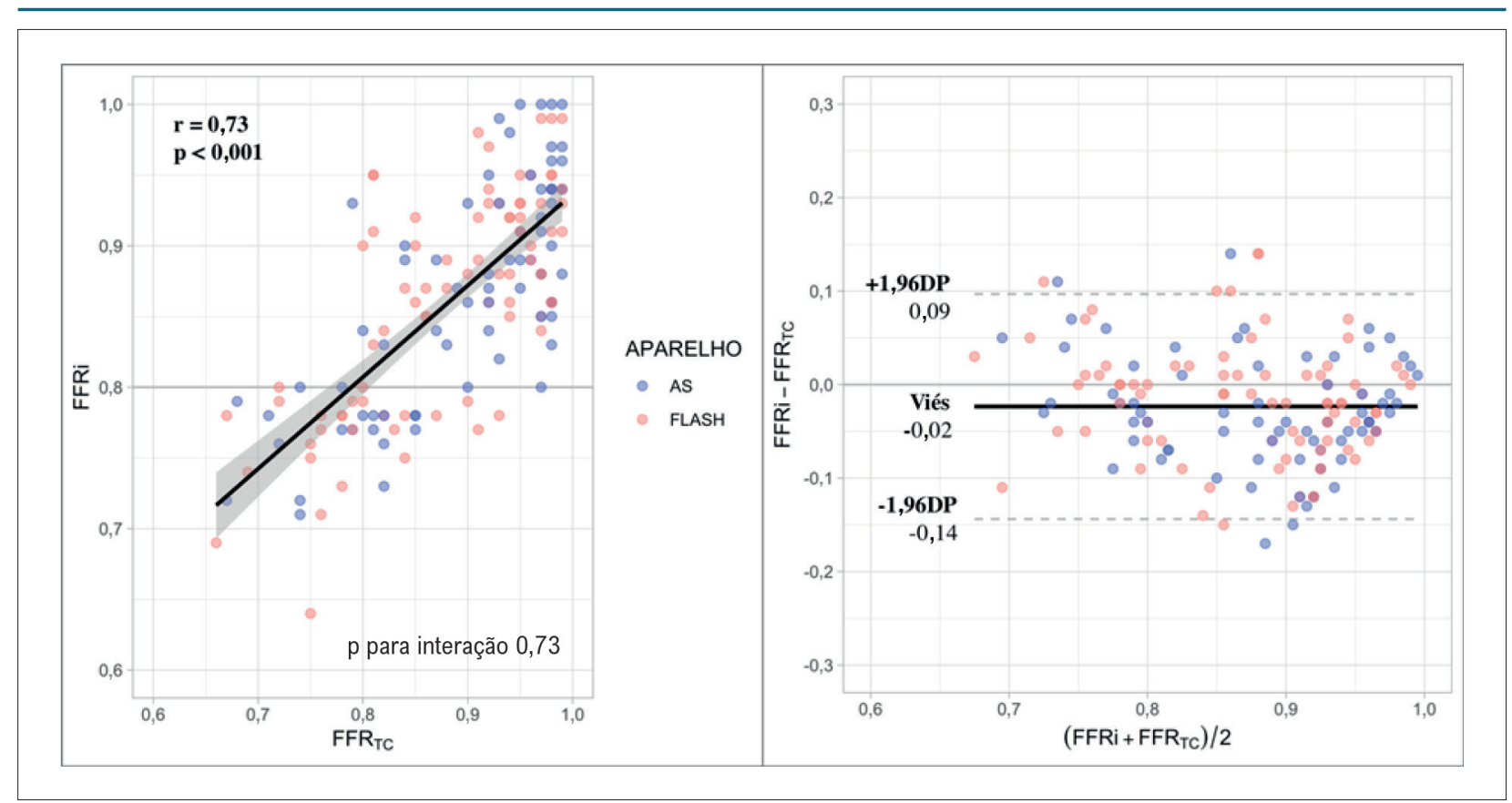

Figura 2 - Correlação (A) e concordância por análise de Bland-Altman (B) entre $F F R_{T C}$ e FFRi (análise por vaso): APARELHO: AS refere-se a tomógrafo de 128 cortes e FLASH, a tomógrafo de 256 cortes.

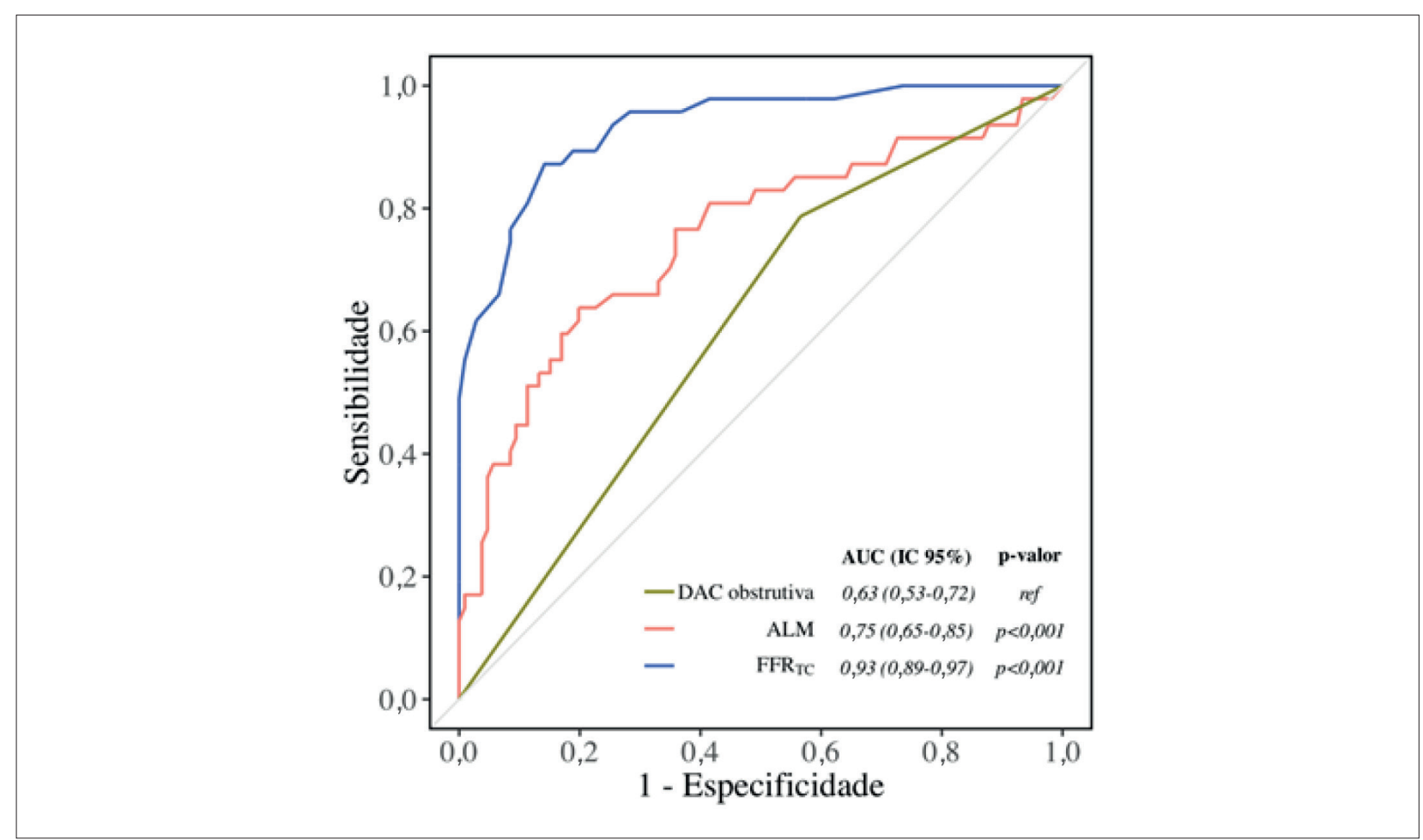

Figura 3 - Performance para o diagnóstico de lesão obstrutiva com limitação de fluxo (FFRi $<0,8)$.

significativa em tronco de coronária esquerda, óstios de coronárias principais ou em bifurcações; oclusões arteriais crônicas; história prévia de cirurgia de revascularização ou implante de stent. Da mesma forma, pacientes com sintomas típicos eventualmente não foram submetidos a exame funcional invasivo (FFRi) por decisão clínica. Portanto, esse estudo deve ser interpretado com a devida atenção ao contexto clínico da população participante (DAC menos grave/complexa e/ou cenários clínicos de maior dúvida diagnóstica). 


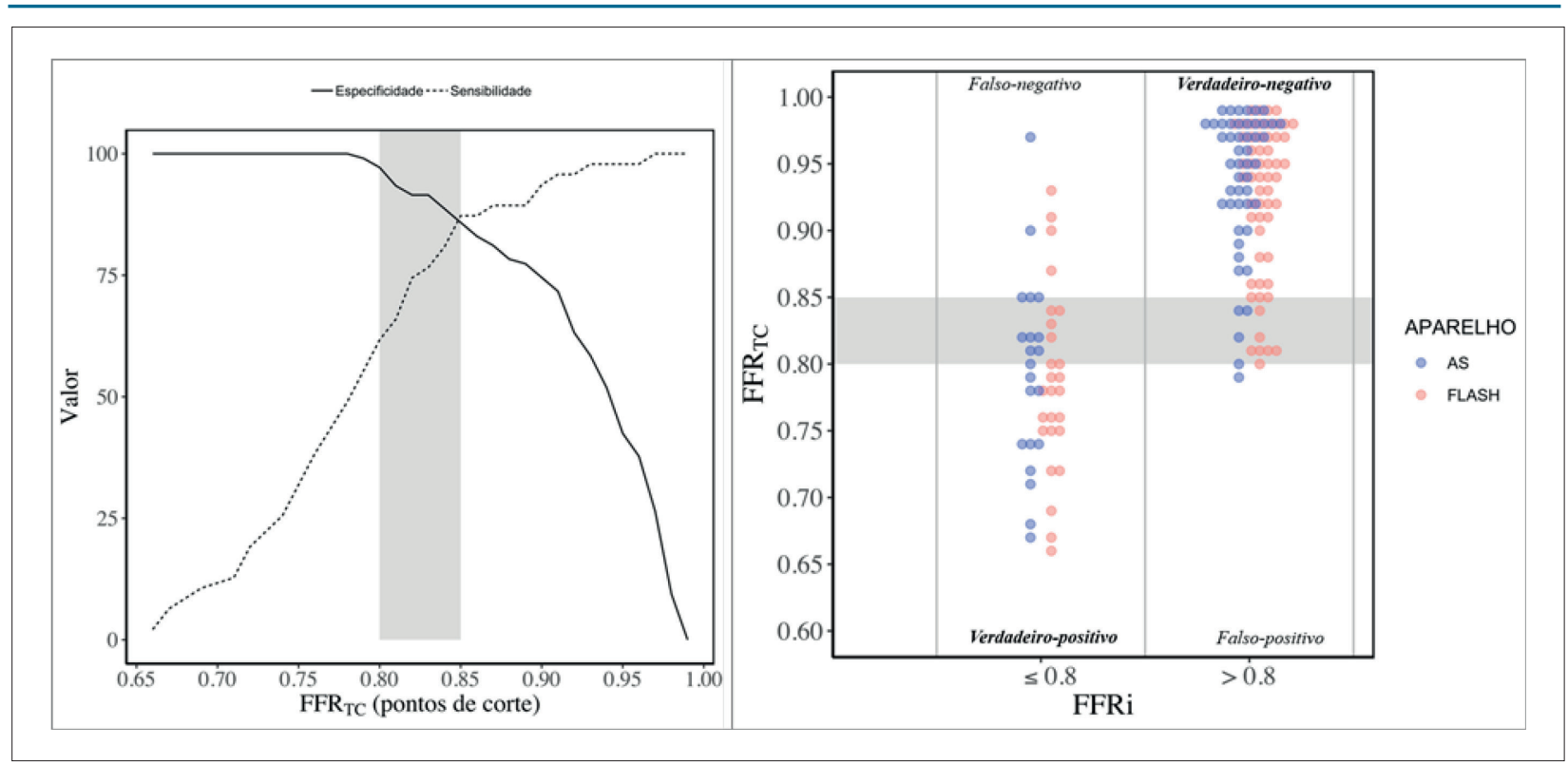

Figura 4 - Performance diagnóstica do valor de $F F R_{T C}<0,85$.

APARELHO: AS refere-se ao tomógrafo de 128 cortes e FLASH, ao tomógrafo de 256 cortes.

\section{Conclusão}

Essa nova versão da $\mathrm{FFR}_{\mathrm{TC}}$, mesmo em tomógrafos de gerações anteriores, apresentou boa performance diagnóstica na detecção de lesões coronarianas obstrutivas com limitação de fluxo, com redução expressiva do número de falsopositivos, o que pode reduzir significativamente a quantidade de pacientes que realizam testes adicionais. A importância clínica desses achados precisa ser validada por estudos especificamente desenhados para avaliações de desfechos clínicos. Esse software apresenta tecnologia inovadora, utilizando aprendizado de máquina, o que possibilita maior acessibilidade, rápida execução e, potencialmente, redução de custos.

\section{Contribuição dos autores}

Concepção e desenho da pesquisa: Magalhães TA, Nomura CH, Ávila LFR, Parga Filho JR; Obtenção de dados: Morais TC, Silva CFG, Paula CB, Torres RA, Magalhães TA; Análise e interpretação dos dados:

\section{Referências}

1. Fihn SD, Blankenship JC, Alexander KP,Bittl JA, Byrne JG, Fletcher BJ, et al. et al. 2014 ACC/AHA/AATS/PCNA/SCAI/STS Focused Update of the Guideline for the Diagnosis and Management of Patients With Stable Ischemic Heart Disease. J Am Coll Cardiol. 2014; 64(18):19291949. doi:10.1016/j. jacc.2014.07.017.

2. Task Force Members, Montalescot G, Sechtem U, Achenbach S, Andreotti F, Arden C, etal. 2013 ESC guidelines on the management of stable coronary artery disease. Eur HeartJ. 2013; 34(38):2949-3003. doi:10.1093/eurheartj/eht296.

3. Cesar LA,Ferreira JF, Armaganyan D, Gowdak LH, Mansur AP, Bodanese LC, et al. Sociedade Brasileira de Cardiologia Diretriz De Doença Coronária Estável. Arq Bras Cardiol. 2014; 103(2 Suppl 2):1-59. doi:10.5935/ abc.2014S004.
Assunção-Jr AN, Dantas Júnior RN, Parga Filho JR; Análise estatística: Assunção-Jr AN, Dantas Júnior RN; Redação do manuscrito: Morais TC, Assunção-Jr AN, Dantas Júnior RN, Magalhães TA, Parga Filho JR; Revisão crítica do manuscrito quanto ao conteúdo intelectual importante: Morais TC, Assunção-Jr AN, Dantas Júnior RN, Nomura $\mathrm{CH}$, Ávila LFR, Parga Filho JR.

\section{Potencial conflito de interesses}

Declaro não haver conflito de interesses pertinentes.

\section{Fontes de financiamento}

O presente estudo não teve fontes de financiamento externas.

\section{Vinculação acadêmica}

Não há vinculação deste estudo a programas de pósgraduação.

4. Little WC, Constantinescu M, Applegate RJ, Kutcher MA,Burrows MT, Kahl FT, et al. Can coronary angiography predict the site of a subsequent myocardial infarction in patients with mild-to-moderate coronary artery disease? Circulation. 1988; 78(5 Pt 1):1157-66.

5. Shaw LJ, Berman DS, Maron DJ, Mancini GB, Hayes SW, Hartigan PM, et al. Optimal medical therapy with or without percutaneous coronary intervention to reduce ischemic burden. Circulation. 2008; 117(10):128391. doi:10.1161/CIRCULATIONAHA.107.743963.

6. Tonino PAL, De Bruyne B, Pijls NHJ, Siebert U, Ikeno F, van Veer M, Klauss $\mathrm{Y}$, et al. Fractional flow reserve versus angiography for guiding percutaneous coronary intervention. N Engl J Med. 2009; 360(3):213-24. doi:10.1056/ NEJMoa0807611. 
7. Nielsen LH, Ortner N, Norgaard BL, Achenbach S, Leipsic J, Abdulla J.et al. The diagnostic accuracy and outcomes after coronary computed tomography angiography vs. conventional functional testing in patients with stable angina pectoris: a systematic review and meta-analysis. Eur Hear J - Cardiovasc Imaging. 2014; 15(9):961-71. doi:10.1093/ehjci/ jeu027.

8. Nørgaard BL, Leipsic J, Gaur S,Seneviratne S, Ko BS, Ito H, et al. Diagnostic performance of noninvasive fractional flow reserve derived from coronary computed tomography angiography in suspected coronary artery disease: The NXT trial (Analysis of Coronary Blood Flow Using CT Angiography: Next Steps). J Am Coll Cardiol. 2014; 63(12):1145-55. doi:10.1016/j.jacc.2013.11.043.

9. Min JK, Leipsic J, Pencina MJ, Berman DS, Kocc BZ, van Mieghem C, et al. Diagnostic accuracy of fractional flow reserve from anatomic CT angiography. JAMA - J Am Med Assoc. 2012; 308(12):1237-45. doi:10.1001/2012.jama.11274.

10. Koo BK, Erglis A, Doh JH, Daniels DV, Jegere S, Kim HS, et al. Diagnosis of ischemia-causing coronary stenoses by noninvasive fractional flow reserve computed from coronary computed tomographic angiograms: Results from the prospective multicenter Discover-Flow (Diagnosis of Ischemia-Causing Stenoses Obtained Via Noni). J Am Coll Cardiol. 2011; 58(19):1989-97. doi:10.1016/j.jacc.2011.06.066.

11. Röther J, Moshage M, Dey D, Schwemmer C, Trobs M, Blachutzik F, et al. Comparison of invasively measured FFR with FFR derived from coronary $\mathrm{CT}$ angiography for detection of lesion-specific ischemia: Results from a PC-based prototype algorithm. J Cardiovasc Comput Tomogr. 2018; 12(2):101-7. doi:10.1016/j.jcct.2018.01.012.

12. Abbara S, Blanke P, Maroules CD,Cheezum M, Choi AD Han BK, et al. SCCT guidelines for the performance and acquisition of coronary computed tomographic angiography: A report of the Society of Cardiovascular Computed Tomography Guidelines Committee Endorsed by the North America. Cardiovasc Comput Tomogr.2016;10(6): 435-49.
13. Leipsic J, Co-Chair F, Abbara S,Achenbach S, Cury R, Earls JP, et al. SCCT Guidelines SCCT guidelines for the interpretation and reporting of coronary CT angiography: A report of the Society of Cardiovascular Computed Tomography Guidelines Committee.J Cardiovasc Comput Tomogr.2014;8(5):342-58. doi:10.1016/j.jcct.2014.07.003.

14. DeLong ER, DeLong DM, Clarke-Pearson DL. Comparing the areas under two or more correlated receiver operating characteristic curves: a nonparametric approach. Biometrics. 1988; 44(3):837-45.

15. WJ Youden. Index for rating diagnostic tests. Cancer. 1950: 32-5.

16. Renker M, Schoepf UJ, Wang R, Meinel FG, Rier JD, Bayer RRnd, et al. Comparison of diagnostic value of a novel noninvasive coronary computed tomography angiography method versus standard coronary angiography for assessing fractional flow reserve. Am J Cardiol. 2014; 114(9):1303-8. doi:10.1016/j.amjcard.2014.07.064.

17. Coenen A, Lubbers MM, Kurata A,Kono A, Dedic A, Chelu RG, etal. Fractional flow reserve computed from noninvasive $C T$ angiography data: diagnostic performance of an on-site clinician-operated computational fluid dynamics algorithm. Radiology. 2015; 274(3):674-83. doi:10.1148/radiol.14140992.

18. Baumann S, Wang R, Schoepf UJ, Steinberg DH, Spearman JV, Bayer RR, etal. etal. Coronary CT angiography-derived fractional flow reserve correlated with invasive fractional flow reserve measurements - initial experience with a novel physician-driven algorithm. Eur Radiol. 2015;25(4):1201-7. doi:10.1007/ s00330-014-3482-5.

19. Wang R, RenkerM, Schoepf UJ,Wichmann JL, Fuller S, Rier J, et al. Diagnostic value of quantitative stenosis predictors with coronary CT angiography compared to invasive fractional flow reserve. Eur J Radiol. 2015; 84(8):150915. doi:10.1016/j.ejrad.2015.05.010.

20. Hadamitzky M, Distler R, Meyer T,Hein F, Kastrati A, Martinoff S, et al. Prognostic value of coronary computed tomographic angiography in comparison with calcium scoring and clinical risk scores. Circ Cardiovasc Imaging. 2011; 4(1):16-23. doi:10.1161/CIRCIMAGING.110.955351. 